ments in which only RNA was required.

34. GenBank accession number M97651. A 1.5-kb Miu I fragment of $A$. castellaniimtDNA [method 1 of (33)]. containing the $3^{\prime}$ terminal region of the LSU rRNA gene, five IRNA genes, and the 5 '-half of the SSU rRNA gene, was subcloned into M13 vectors [J. Messing, Methods Enzymol. 101, 20 (1983)] from a $6.7-\mathrm{kb}$ Bam $\mathrm{HI}$ insert that had been cloned initially into PAT153 [A. J. Twigg and D. Sherratt, Nature 283, 216 (1980). A nested deletion series was generated [S. Henikoff, Gene 28, 351 (1984)] and both strands of the DNA were sequenced $[F$. Sanger, S. Nicklen, A. R. Coulson, Proc. Natl. Acad. Sci. U.S.A. 74, 5463 (1977); Sequenase, version 2.0 (U.S. Biochemicals, Cleveland. $\mathrm{OH}$ )] For some experiments (Figs. 4 and 5), tRNA gene specific oligonucleotides, $5^{\prime}$ end-labeled with ${ }^{32} \mathrm{P}$, were used as sequencing primers.

35. After RNase $A$ treatment, nDNA and mtDNA [method 2 of (33)] were hydrolyzed with restric tion endonucleases, and the products $(2.5 \mu \mathrm{g}$ of nDNA and $2.5 \mathrm{ng}$ of miDNA per lane) were separated by electrophoresis in a $0.7 \%$ agarose gel in $40 \mathrm{mM}$ tris acetate and $1 \mathrm{mM}$ EDTA- $\mathrm{Na}_{2}(\mathrm{pH}$ 8.1 ) for 2 hours at $150 \mathrm{~V}$. DNA was transferred to GeneScreen Plus nylon membrane (DuPont Canada, Lachine, Quebec) according to the manufacturer's specifications. Before addition of probe the blot was hybridized for 2 hours at $65^{\circ} \mathrm{C}$ in 30 $\mathrm{ml}$ of $5 \times$ saline sodium citrate (SSC; $1 \times$ is $0.15 \mathrm{M}$ $\mathrm{NaCl}$ and $0.015 \mathrm{M}$ sodium citrate), $20 \mathrm{mM}$ sodium phosphate (pH 7.0), 10x Denhardt's solution [1. Maniatis, E. F. Fritsch, J. Sambrook, Molecular Cloning: A Laboratory Manual (Cold Spring Har. bor Laboratory, Cold Spring Harbor, NY, 1982), p. 327 ], $7 \%$ SDS, and $10 \%$ dextran sulfate. Overnight hybridization was with the addition of $100 \mathrm{ng}$ of oligonucleotide probe, $5^{\prime}$ end-labeled with [ $\gamma$-32P]adenosine triphosphate (ATP) by T4 polynucleotide kinase. The hybridization temperature was $6^{\circ}$ to $7^{\circ} \mathrm{C}$ lower than the calculated dissociation temperature $\left(T_{d}\right)$ of the oligonucleotide probe [R. A. Zeff and J. Geliebter, Focus 9 (no. 2), (1987)]. The blot was washed for a total of $60 \mathrm{~min}$ in $2 \times$ SSC and $1 \%$ SDS and for $15 \mathrm{~min}$ in $0.5 x$ SSC and $0.1 \%$ SDS at a wash temperature $7^{\circ}$ to $12^{\circ} \mathrm{C}$ below $T_{d}$. For reprobing experiments, the blot was stripped of bound oligonucleotide by incubation in $1 \times \mathrm{SSC}$ at $80^{\circ} \mathrm{C}$ for $10 \mathrm{~min}$.

36. Primer extension analysis and RNA sequencing were performed according to a published protocol [J. Geliebter, Focus 9 (no. 1), 5 (1987)] but without actinomycin D. Mitochondrial nucleic acids were treated with DNase I, and the resulting RNA $(10 \mu \mathrm{g})$ was annealed to $5 \mathrm{ng}$ of $5^{\prime} 32 \mathrm{p}$-endlabeled oligonucleotide (23) that served as a primer for extension by avian myeloblastosis virus (AMV) reverse transcriptase in the presence of deoxynucleoside triphosphates and either the presence (G, $A, T$, and $C$ ) or absence (E) of dideoxynucleoside triphosphates (ddNTPs) (Figs. 4 and 5). In some experiments, we used terminal deoxynucleotidyl transferase to eliminate se quence ambiguities resulting from the interna pausing of reverse transcriptase [D. C. DeBorde C. W. Naeve, M. L. Herlocher, H. F. Maassab, Anal. Biochem. 157, 275 (1986)]. For tRNAMet1 and tRNAMet2 (Fig 4 A and D), the products of standard RNA sequencing reactions were extracted with phenol, precipitated three times with ethanol dissolved in water, and heated at $80^{\circ} \mathrm{C}$ for $2 \mathrm{~min}$ Samples were then cooled on ice and incubated with $40 \mathrm{U}$ of terminal deoxynucleotidyl transferase (Promega) in the presence of $1 \mathrm{mM}$ dATP, $100 \mathrm{mM}$ cacodylic acid (pH 6.8), $1 \mathrm{mM} \mathrm{CoCl}, 0.1 \mathrm{mM}$ dithiothreitol, and bovine serum albumin $(0.1 \mathrm{mg}$ $\mathrm{ml}$ ) in a $20-\mu \mathrm{l}$ reaction volume at $37^{\circ} \mathrm{C}$ for $60 \mathrm{~min}$ Samples were precipitated with ethanol before we loaded them onto the sequencing gel.

37. Supported by Medical Research Council of Canada grant MT-4124 (M.W.G.), a Walter P. Sumner Memorial Fellowship (K.M.L.), and a fellowship (M.W.G.) from the Canadian Institute for Advanced Research.

10 August 1992; accepted 13 November 1992

\title{
Tumor Suppression in Xiphophorus by an Accidentally Acquired Promoter
}

\author{
Dieter Adam, ${ }^{*}$ Nicola Dimitrijevic, Manfred Schart।
}

Melanoma formation in the teleost Xiphophorus is caused by a dominant genetic locus, Tu. This locus includes the Xmrk oncogene, which encodes a receptor tyrosine kinase. Tumor induction is suppressed in wild-type fish by a tumor suppressor locus, $R$. Molecular genetic analyses revealed that the $T u$ locus emerged by nonhomologous recombination of the Xmrk proto-oncogene with a previously uncharacterized sequence, $D$. This event generated an additional copy of $X m r k$ with a new promoter. Suppression of the new Xmrk promoter by $\boldsymbol{R}$ in parental fish and its deregulation in hybrids explain the genetics of melanoma formation in Xiphophorus.

Melanoma formation in Xiphophorus has been studied since 1922 (1). Crossing of a tumor-free wild-type strain carrying $T u$ and $R$ with a strain carrying neither locus produces progeny that develop tumors. The emergence of this phenotype is due to the loss of R-bearing chromosomes in the hybrids, which allows expression of the full oncogenic potential of $\mathrm{Tu}(2)$. We have addressed how such a potentially injurious locus was generated and maintained during evolution and how its transforming activity is suppressed in the feral populations.

We recently showed that Xmrk, a gene encoding a receptor tyrosine kinase closely related to the epidermal growth factor (EGF) receptor, is the critical constituent of the $T u$ locus (3). Xmrk is present in the Xiphophorus genome in two different copies. All examined specimens of the genus $X_{i-}$ phophorus and related species contain one $\mathrm{X}$ mrk gene that fulfills all the characteristics of a proto-oncogene $(4,5)$. A second copy of Xmrk is present only in individuals that carry a $T u$ locus. This second copy is a structural constituent of the $T u$ locus (3-5) and is the melanoma-inducing oncogene. The two genes reside within 2 centimorgans (cM) of each other on the sex chromosomes (5), with X- and Y-chromosomal alleles of the oncogene (4).

The proto-oncogene is differentially expressed during embryogenesis and is expressed at low levels in adults in normal epithelial tissues as well as in melanoma. Its expression is independent of $R$. The oncogene, however, is only expressed in melanoma (at high levels) and only when $R$ is absent $(3,4,6)$. Primer extension analyses

D. Adam, Genzentrum, Max-Planck-Institut für Biochemie, D-8033 Martinsried, Germany.

N. Dimitrijevic and M. Schartl, Physiologische Chemie I. Theodor-Boveri-Institut für Biowissenschaften (Biozentrum) der Universität Würzburg, D-8700 Würzburg. Germany, and Genzentrum, Max-Planck-Institut für Biochemie, D-8033 Martinsried, Germany.

-To whom correspondence should be addressed, at the Department of Molecular Biology. Signal Trans. duction Laboratory. Bristol-Myers Squibb Pharmaceutical Research Institute, Princeton, NJ 08543. have revealed that the Xmrk oncogene and proto-oncogene have different transcription start sites $(4,7)$ and that their transcripts differ in size by $\sim 1 \mathrm{~kb}(4)$. These observations indicate that there may be differencesi in transcriptional control between the, Xmrk oncogene and proto-oncogene, possibly because of structural differences in the 5 ' promoter region.

To investigate this potential structural, difference, we cloned a full-length cDNA corresponding to the $\mathrm{Xmrk}$ proto-oncogene from the Xiphophorus embryonal epithelial cell line A2 (8). Alignment of the clone with the previously isolated oncogene cDNA (3) revealed colinearity of the sequences starting $3^{\prime}$ of codon 10 in exon 1 (Fig. 1). The two sequences had no similarity $5^{\prime}$ of this region, however. This observation confirmed that the Xmrk oncogene and proto-oncogene had different $5^{\prime}$ regions and thus possibly also different promoters and regulatory sequences.

To isolate the respective promoter regions, we first screened a genomic library of Xiphophorus maculatus with a DNA fragment comprising the $5^{\prime}$ end of the oncogene $\mathrm{cDNA}$. A 5.3-kb clone $(\lambda \times 21-1)$ was isolated. This clone contained exon 1 of the Xmrk oncogene as well as 5' flanking sequences with TATA- and CAAT-like elements located at the expected distance from the transcription start site (Fig. 1). Surprisingly, the potential promoter se-

Table 1. Expression of the Xmrk oncogene promoter in the absence (PSM cells) or presence (A2 cells) of the $R$ locus. CAT conversion rates obtained with the oncogene promoter are shown relative to the basal CAT conversion rates seen with the internal standards in two independent experiments (25).

\begin{tabular}{clc}
\hline Experiment & Cells & CAT activity \\
\hline 1 & PSM & 8.96 \\
\multirow{2}{*}{2} & A2 & 1.42 \\
& PSM & 36.28 \\
& A2 & 5.56 \\
\hline
\end{tabular}


quence of the oncogene resembled the promoters of "nonhousekeeping genes" (9). It did not contain the GC-rich sequences driving transcription of the closely related human EGF receptor gene $(10)$ or the rat neu gene (11). We then used a probe specific for the 5 ' end of the proto-oncogene $\mathrm{cDNA}$ to isolate a genomic clone that contained sequences identical to the $5^{\prime}$ untranslated region of the proto-oncogene cDNA ( $\lambda E 5)$.

Participation of the $R$ locus in transcriptional regulation was suggested by the observation that the Xmrk oncogene is overexpressed in melanomas of hybrid fish that do not carry $R$ (4). To determine whether transcription from the oncogene promoter was dependent on the absence of $R$, we cloned the putative $5^{\prime}$ regulatory region, including the transcription start site and 10 bp of $5^{\prime}$ untranslated leader sequence, into plasmid pBLCAT3 upstream of the chloramphenicol acetyltransferase (CAT) reporter gene. The plasmid was then transfected into A2 cells, which are derived from an R-bearing Xiphophorus genotype (8), and into PSM cells, a cell line established from malignant melanoma of hybrids that do not carry the $R$ locus (12).

The activity of the oncogene promoter fragment was approximately six times higher in PSM cells as compared with A2 cells (Table 1). Previous studies with promoter sequences unrelated to the oncogene promoter had shown a comparable transcriptional activity in the two cell lines (13). Therefore, the elevated activity of the oncogene promoter was not simply due to a generally higher rate of transcription in PSM cells but rather suggests that the $R$ locus negatively regulates the oncogene promoter in A2 cells. However, although expression of the Xmrk oncogene is not detectable in nontumorous tissues of Xiphophorus (4), we observed a basal level of CAT activity in the $A 2$ cells. We suggest that there are other $5^{\prime}$ regulatory elements outside the cloned oncogene promoter fragment that provide additional suppression of the Xmrk oncogene in parental feral fish.

To verify the divergent organization of the Xmrk oncogene and proto-oncogene 5' regions, we hybridized DNA fragments containing the $5^{\prime}$ region of each gene to genomic DNA from $X$. maculatus carrying the proto-oncogene plus an $X$ - or $Y$-chromosomal allele of the oncogene. As expected, the two probes recognized distinct DNA fragments (Fig. 2). The proto-oncogene probe hybridized uniformly to an 8.4-kb band in all genotypes. The oncogene probe hybridized to a 5.3-kb band in genotypes with a Y-chromosomal Xmrk oncogene (as expected from $\lambda \times 21-1$; see Fig. $3 B$ ) and to a $5.6-\mathrm{kb}$ band in genotypes with the $\mathrm{X}$-chromosomal form of the Xmrk onco- gene. The dual hybridization of the oncogene probe is most likely due to minor polymorphisms in the restriction pattern of the two oncogenes. The production of distinct hybridization patterns by the oncogene and proto-oncogene probes confirms that the two genes have distinct $5^{\prime}$ regions at the genomic level.

Surprisingly, the oncogene probe also hybridized strongly to a $2.3-\mathrm{kb}$ band. This 2.3-kb fragment is present in all Xiphophorus genotypes tested so far, regardless of whether the $T u$ locus is present $(n=30)$ or absent $(n=13)$. The fragment was cloned and shown to be almost identical in sequence to the oncogene promoter from its $5^{\prime}$ end to the transcription start site (Fig. 1). No substantial sequence similarity to either the Xmrk oncogene or proto-oncogene was apparent $3^{\prime}$ of this site; however, a single, long open reading frame begins 11 nucleotides $3^{\prime}$ of the Xmrk oncogene homology region and extends for $>500$ nucle- : otides. We designated this locus $D$.

To explain the sequence similarity of the

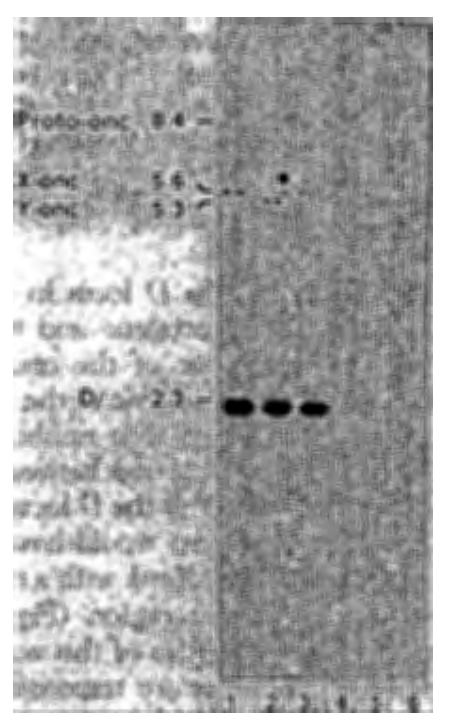

Fig. 2. Different genomic structures of the Xmrk proto-oncogene and oncogene $5^{\prime}$ regions. Southern blot analyses of Xiphophorus genomic DNA were performed with probes corresponding to the $5^{\prime}$ region of the Xmrk oncogene (lanes 1 to 3 ) and the $5^{\prime}$ region of the Xmrk proto-oncogene (lanes 4 to 6 ). Fragments specific for each $X m r k$ locus are indicated (Protoonc, proto-oncogene; $X$-onc, $X$-chromosomal oncogene; $Y$-onc, $Y$-chromosomal oncogene), with sizes in kilobases. Faint bands of approximately $6.5 \mathrm{~kb}$ are sequences that cross-hybridize even under conditions of high stringency; these bands do not cosegregate with the Xmrk loci (21), however, and are therefore most likely located on other chromosomes. The 2.3-kb band is a fragment of the $D$ locus. Eco RIdigested genomic DNA ( $X$. maculatus stock Rio Jamapa, $X^{\text {Tu-Sd }} X^{\text {Tu-Sd }}$, lanes 1 and $4 ; Y^{\text {Tu-Sr} /}$ $Y^{T u-S r}$, lanes 2 and $5 ; \dot{X}^{T u-S P} / X^{T u-S P}$, lanes 3 and 6) was analyzed (22). 
Fig. 3. Model for evolution of the Xiphophorus genes involved in melanoma formation. Genes are represented by rectangles, and the recombination event is represented by the dashed arrow. Potential negative regulation by the Rlocus is indicated. PI. promoter of the Xmrk proto-oncogene; $\mathrm{PD}$, promoter of the $D$ locus. Relevant genomic clones of the Xmrk loci and the $D$ locus are indicated by horizontal brackets. $X, X$-chromosome; Y, Y-chromosome. (A) A nonhomologous recombination translocated the Xmrk proto-oncogene to a new location $2 \mathrm{cM}$ distant, fusing it to the promoter of the $D$ locus.

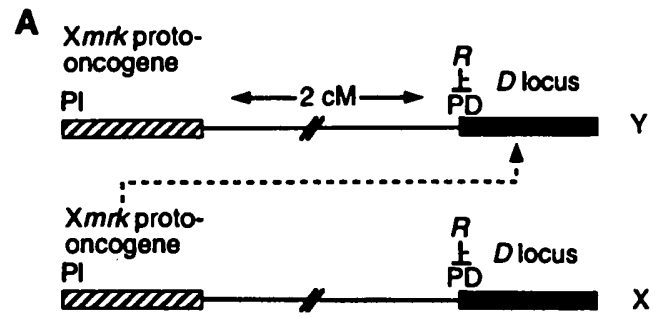

B Xmrk proto-

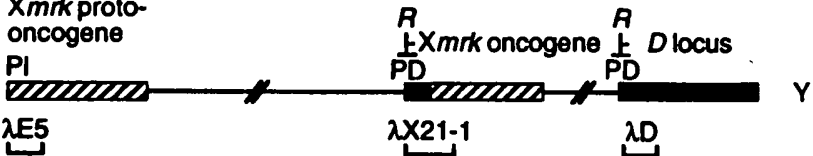

C

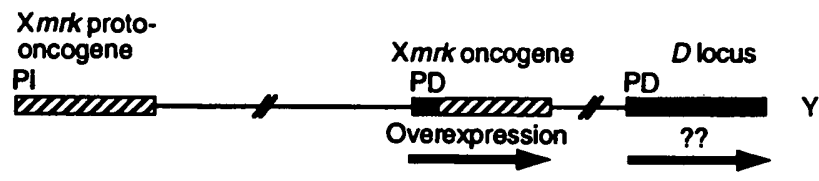

This event generated an ancestral Xmrk oncogene, most likely the Y-chromosomal form (23). The truncated $X$-chromosome was lost during meiosis. The $X$-chromosomal form of the Xmrk oncogene (not shown) diverged from the ancestral form at a later stage by homologous recombination between the $Y$ - and $X$-chromosomes (23). (B) Present situation in wild-type $X$. maculatus (shown only for the $Y$-chromosome). The Xmrk oncogene is controlled by its new promoter (regulated by $R$ ). which prevents its oncogenic action. (C) Present situation in hybrids of Xiphophorus that do not carry the $R$ locus. The Xmrk oncogene is overexpressed from its "acquired" promoter, resulting in melanoma formation. Expression of the Xmrk proto-oncogene remains unaffected. It remains to be elucidated whether expression of the $D$ locus changes in response to loss of the $R$ locus.

Xmrk oncogene and the $D$ locus in the 5 region of the Xmrk oncogene and the almost identical sequence of the oncogene and the $\mathrm{Xmrk}$ proto-oncogene in the region downstream, we suggest that nonhomologous recombination occurred between the Xmrk proto-oncogene and the $D$ locus (Fig. $3 \mathrm{~A})$. This rearrangement would have generated a second copy of Xmrk with a new, $D$ locus-derived promoter region (Fig. 3B). Presumably, the properties of this accidentally acquired promoter are responsible for the overexpression of Xmrk in pigment cells of hybrid genotypes that do not carry the $R$ locus (Fig. 3C).

We suggest that the product of the $R$ locus participates either directly or indirectly in transcriptional control of the Xmrk oncogene promoter. It is possible that the $D$ locus represents the physiologic target for $R$, so that in essence the tumor suppressor function of $R$ would be an accidental "side effect" of the regulation $R$ exerts on $D$. However, this accidental suppression of the Xmrk oncogene has ensured the evolutionary survival of the oncogene. An actively transcribed Xmrk oncogene would have generated melanoma in the first animal carrying the recombinant $X_{m r k}$ gene, thereby eliminating the oncogene from the population.

The sequence similarity between the
Xmrk proto-oncogene and oncogene terminates in the region encoding the signal peptide, which is cleaved off in the mature $\mathrm{Xmrk}$ receptor tyrosine kinases. Hence, the original oncogene and proto-oncogene products may have been essentially identical. Over the course of evolution, the oncogene may have acquired mutations that further enhanced its tumorigenicity in hybrid fish that do not carry the $R$ locus.

The Xmrk oncogene contains a stretch of sequence (framed by a large box in Fig. 1) that is present neither in the $D$ locus nor in the Xmrk proto-oncogene. This sequence encompasses the entire untranslated $5^{\prime}$ region of the oncogene transcript, the ATG codon, and the codons responsible for the new but obviously functional signal peptide. Polymerase chain reaction (PCR)mediated amplification of this sequence from DNA of Xiphophorus genotypes carrying either the Xmrk proto-oncogene alone or both proto-oncogene and oncogene yielded identical products in every case (Fig. 4). This result suggests that another locus has contributed this sequence to the oncogene locus, perhaps through a second recombination event. The fact that this fragment is flanked by an 8-nucleotide direct repeat (Fig. 1) is consistent with this hypothesis.

To demonstrate that the presence of the

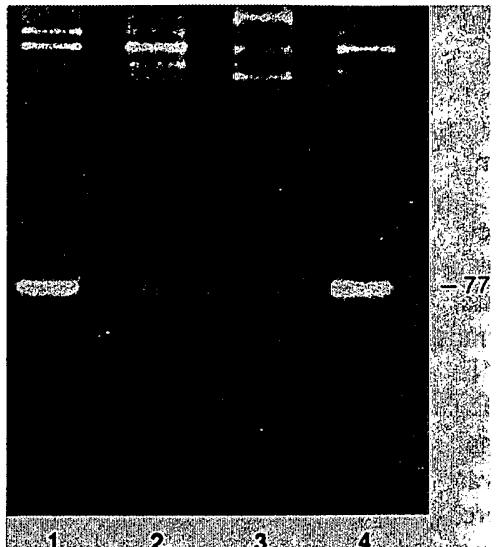

Fig. 4. Amplification of the Xmrk oncogene promoter sequence that is not derived from either the Xmrk proto-oncogene or the $D$ locus PCR was performed (24) on genomic DNA from fish carrying X-chromosomal (Tu-Sp) or Y-chromosomal (Tu-Si) alleles (or both) of the Xmrk oncogene (lanes 1 and 4 ) or from fish that carry only the Xmrk proto-oncogene (lanes 2 and 3 ) Similar results were obtained with nine othe genotypes. DNA samples were obtained from $X$. maculatus (lanes 1,2 , and 4 ) and $X$. helleri (lane 3). The size of the fragments (in base pairs) is indicated in the right margin

new Xmrk oncogene promoter was not limited to the genotypes and cell lines used for this study, we performed PCR analyses on a variety of genotypes, using primers that flank the entire region of recombination. The expected PCR product was obtained from all genotypes that carried an Xmrk oncogene but not from genotypes without an Xmrk oncogene (21).

In summary, these data explain how a potentially deleterious gene, the Xmrk oncogene, was generated and has been maintained over many generations in the germ line of Xiphophorus fish. Through transcriptional control of its accidentally acquired promoter, the oncogene remains inactive until the factor (or factors) that suppress its expression in the melanophore cell lineage become impaired in the hybrid genome. Because the original site of action for the $R$ locus was most likely not the Xmrk gene, the situation in Xiphophorus may best be described as "tumor suppression by accident."

\section{REFERENCES AND NOTES}

1. A. W. Bellarmy, Anat. Rec. 23, 98 (1922); M. Gordon Genetics 12, 253 (1927); C. Kosswig. Z. Induk Abstamm. Verertungsl. 47, 150 (1928); K. D. Kallman and J. W. Atz, Zoologica 51, 107 (1966).

2. M. R. Ahuja and F. Anders, Prog. Exp. Tumor Res. 20, 380 (1976)

3. J. Wittbrodt et al., Nature 341, 415 (1989).

4. D. Adam, W. Mäueler, M. Schartl, Oncogene 6, 73 (1991)

5. M. Schartl, Genetics 126, 1083 (1990)

6. W. Mäueler, F. Raulf, M. Schartl, Oncogene 2. 421 (1988). 
7. D. Adam, unpublished data.

8. C. Kuhn, U. Vielkind et al., In Vitro 15, 537 (1979).

9. W. S. Dynan. Trends Genet. 2, 196 (1986).

10. S. Ishii et al., Proc. Natl. Acad. Sci. U.S.A. 84, 4374 (1987).

11. T. Suen and M. Hung. Mol. Cell. Biol. 10, 6306 (1990).

12. Y. Wakamatsu, Cancer Res. 41, 679 (1981).

13. H. Friedenreich and M. Schartl, Nucleic Acids Res. 18, 3299 (1990).

14. A. P. Feinberg and B. Vogelstein, Anal. Biochem. 132, 6 (1983).

15. J. Devereux, P. Haeberli, O. Smithies, Nucleic Acids Res. 12, 387 (1984)

16. The proto-oncogene cDNA was isolated from an oligo(dT)-primed library prepared by standaro methods with polyadenylated [poly $\left.(A)^{+}\right]$RNA from the Xiphophorus embryonal epithelial cell line A2 (8). The $D$ locus and $X$ mrk oncogene promoter clones were isolated from genomic sublibraries ( $X$. maculatus stock Rio Jamapa $X^{\text {Tu-Sd) }}$ $Y^{\text {Tu-SY }}$ constructed as described (3), except that DNA fragments of 1.5 to $2.5 \mathrm{~kb}$ ( $D$ locus clone) and 4 to $6 \mathrm{~kb}$ (oncogene) were used for ligation. Probes were labeled by random priming (14). The A2 CDNA library was screened with probe 3-2E (3), and the Xmrk oncogene sublibrary was screened with a 544-bp Eco RI-Eco RV fragment of 3-2E ( $5^{\prime}$ end of the CDNA). The $D$ locus sublibrary was screened with a PCR fragment comprising nucleotides 1 to 431 of the Xmrk oncogene. The primers used for the amplification were DA10 (5'-GAATTCCCCAGACCTGT TTGTGTTGGAGG-3', position 1 to 29 of the oncogene sequence) and DA11 (5'-CCTTTCTGTCCGGGTCTGTGCTGCAGCAG-3', position 402 to 431 of the oncogene sequence). The PCR reaction contained $200 \mu \mathrm{M}$ deoxynucleotide triphosphate, 100 pmol of each primer, $1 \mathrm{ng}$ of template DNA and $0.5 \mathrm{U}$ of Taq DNA polymerase (Amersham) in $\mathbf{5 0}$ $\mu /$ of $1 \times$ reaction buffer (Amersham). Amplifications were carried out for 35 cycles $\left(92^{\circ} \mathrm{C}\right.$ for $60 \mathrm{~s}$, $60^{\circ} \mathrm{C}$ for $60 \mathrm{~s}$, and $72^{\circ} \mathrm{C}$ for $90 \mathrm{~s}$ ). Hybridizations were done at $42^{\circ} \mathrm{C}$ in $50 \%$ formamide, $5 \times$ standard saline citrate (SSC), and $1 \%$ SDS, and washes were done at $68^{\circ} \mathrm{C}, 0.1 \times \mathrm{SSC}$, and $1 \%$ SDS. The BESTFIT, GAP, and WORDSEARCH alignments of the GCG package (15) were used to determine sequence homologies.

17. B. Luckow and G. Schütz, Nucleic Acids Res. 15. 5490 (1987).

18. W. Mäueler et al., Oncogene 3, 113 (1988)

19. C. Steiner and C. Kaltschmidt, Trends Genet. 5 , 138 (1989).

20. C. M. Gorman. L. F. Moffat, B. H. Howard, Mol. Cell. Biol. 2. 1044 (1982)

21. D. Adam, N. Dimitrijevic, M. Schartl, unpublished data.

22. The 5 ' region of the $\dot{X} m r k$ proto-oncogene was amplified in a PCR reaction containing primers ND1 (5'-GCAGCTCGAGCAGCGCCATTATTACTGTC-3', position 215 to 188 of the protooncogene cDNA sequence) and ND2 (5'. GACTTGCGCCGAGCCAGCAGACAGGCAGGACCC-3', position 6 to 37 of the proto-oncogene cDNA sequence). The $5^{\prime}$ region of the Xmrk oncogene was amplified in a PCR reaction containing primers DA10 and DA11 (16). The PCA products were separated on $2 \%$ agarose gels, purified, and labeled by random priming. Southern hybridizations were performed as described (3), except that the filters were washed at $72^{\circ} \mathrm{C}$ in $0.1 \times$ SSC and $1 \%$ SDS.

23. M. SchartI and D. Adam, Pigm. Cell Res. 2 (suppl.), 173 (1992)

24. The primers used in the PCR reactions were OP1 (5'-ATGGAGCAGCAGTCTGACCTG-3', position 290 to 310 of the oncogene sequence) and OP2 (5'-CCGCTCCTCCGCGCAGAAAC-3', position 347 to 365 of the oncogene sequence), which border the region framed by the large box in Fig. 1.

25. The $X m r k$ oncogene promoter was cloned into PBLCAT3 (17). Orientation and cloning sites were confirmed by sequencing. The sequence fused to the CAT gene spans the region from position 1 to 288 of the oncogene sequence (see Fig. 1). A detailed description of cell lines A2 and PSM is given elsewhere (18). DNA transfections were performed in triplicate according to (13) with an automated precipitator (19). CAT activity was measured as described (20), with the following modifications. Cells were lysed by three freezethaw cycles, and after centrifugation the protein concentration in the supernatant was determined (Protein Dye Reagent, Biorad). Identical amounts of protein were incubated overnight with $\left[{ }^{14} \mathrm{C}\right.$ )chloramphenicol and acetyl coenzyme $A$ The acetylated derivatives were separated from the unacetylated form by thin-layer chromatography (TLC), and the TLC plates were subsequently autoradiographed. CAT conversion rates were determined with a scintillation counter. For experiment 1, pBLCAT3 was used as an internal standard; for experiment 2 , the internal standard was
pBLCAT3 containing a DNA fragment from the intron of $\lambda \times 21-1$ instead of the oncogene promoter. In both experiments, the low basal CAT con version values obtained with the internal standards were set to " 1 " relative to the values obtained with the oncogene promoter.

26. We thank B. Wilde, S. Hölter, and J. Duschl for technical assistance and J. B. Bolen for discus sions and critical comments on the manuscrip This work was supported by grants to M.S from the Bundesministerium für Forschung und Technologie through "Schwerpunkt: Grundlagen und Anwendungen der Gentechnologie," the Deutsche Forschungsgemeinschaft through "Schwerpunkt: Klassische und Molekulare Tumorzytogenetik," and the Max-Planck-Gesellschaft.

27 August 1992; accepted 16 November 1992

\title{
Multiple Output Channels in the Basal Ganglia
}

\author{
John E. Hoover and Peter L. Strick*
}

The neural circuits that link the basal ganglia with the cerebral cortex are critically involved in the generation and control of voluntary movement. Retrograde transneuronal transport of herpes simplex virus type 1 was used to examine the organization of connections in the cebus monkey between an output nucleus of the basal ganglia, the internal segment of the globus pallidus (GPi), and three cortical areas: the primary motor cortex, the supplementary motor area, and the ventral premotor area. Spatially separate regions of the GPi were labeled after virus injections into each cortical area. The GPi projects to multiple cortical motor areas, and this pallidal output is organized into discrete channels. This information provides a new anatomical framework for examining the function of the basal ganglia in skeletomotor control.

The basal ganglia are subcortical brain nuclei that are critical for the central generation and control of voluntary movement. It has been suggested that these structures are involved in the internal generation of movement, the automatic execution of motor plans, and the acquisition and retention of motor skills (1). Dysfunction of the basal ganglia, as occurs in Parkinson's disease, is associated with striking disorders of movement (2).

The input nuclei of the basal ganglia (that is, the caudate and putamen) receive substantial projections from diverse regions of the cerebral cortex, including motor, sensory, prefrontal, and limbic cortical areas. The output nuclei of the basal ganglia (that is, the GPi and the substantia nigra pars reticulata) send their axons to the thalamus and, by this route, project back upon the cortex. Thus, a major aspect of basal ganglia circuitry is its participation in multiple open and closed loops with the cerebral cortex (3).

Our understanding of the organization of basal ganglia loops with the cerebral cortex has evolved considerably over the last 20

Research Service, Veterans Administration Medica Center and Departments of Neurosurgery and Physiology, State University of New York Health Science Center, Syracuse, NY 13210.

"To whom correspondence should be addressed. years. In the past, the output of the basal ganglia was thought to terminate in a single region of the thalamus and to influence a single cortical area, the primary motor cortex (4). According to this view, the basal ganglia funneled information from widespread regions of the cerebral cortex into the motor system. It is now clear that the output of the basal ganglia terminates in thalamic regions that gain access to a wider region of the frontal lobe than previously suspected (5). Indeed, five parallel basal gangliathalamocortical loops have been identified, each of which is focused on a particular region of the frontal cortex (6). These loops were designated the skeletomotor, oculomotor, dorsolateral prefrontal, lateral orbitofrontal, and anterior cingulate circuits. We have now examined the organization of the skeletomotor circuit with a neuroanatomical technique that makes use of retrograde transneuronal transport of herpes simplex virus type 1 (HSV-1) (7). This technique provides a unique method for labeling a chain of synaptically linked neurons. Our results provide evidence for at least three skeletomotor circuits, each of which involves a spatially separate region of GPi and influences a different cortical motor area.

We injected the McIntyre-B strain of HSV - 1 into the arm representations of either the primary motor cortex, the supple- 